American Journal of Infectious Diseases 8 (2): 106-111, 2012

ISSN 1553-6203

(C) 2012 Science Publications

\title{
Impact of Antimicrobial Stewardship Programme on Carbapenem Resistance in Gram Negative Isolates in an Indian Tertiary Care Hospital
}

\author{
${ }^{1}$ Namita Jaggi, ${ }^{2}$ Pushpa Sissodia and ${ }^{2}$ Lalit Sharma \\ ${ }^{1}$ Department of Lab Services and Infection Control, \\ Artemis Health Institute, Sector 51, Gurgaon, Haryana \\ ${ }^{2}$ Department of Lab Services, Artemis Health Institute, Sector 51, Gurgaon, Haryana
}

\begin{abstract}
Problem statement: Increasing Antimicrobial resistance in the World is constantly becoming a Global threat and there is an urgent need to prevent its spread. Various studies of last decade have shown reduced trends of antimicrobial resistance in the pathogens as an outcome of the Antimicrobial Stewardship Programs. In view of this, the present four years' study was carried out to analyse the impact of Antimicrobial Stewardship Programs on carbapenem resistance in Gram negative isolates in a Tertiary care hospital in India. It involved a retrospective analysis of carbapenem resistance in Gram negatives for one year (July 2007 to June 2008), followed by prospective evaluation of the impact of stewardship interventions on resistance patterns (July 2008 to Jun 2011). Approach: Our study was staged into four parts: (1) July 2007 to June 2008: Resistance patterns of Gram negative isolates-E.coli, Klebsiella, Pseudomonas and Acinetobacter baumannii towards carbapenems were studied. (2) July 2008: Phase I intervention programme Implementation of an antibiotic policy in the hospital. (3) July 2008 to June 2010: The Impact of Phase I intervention programme was assessed subsequently. (4) July 2010 to June 2011: Phase II intervention programme: Formation and effective functioning of the antimicrobial stewardship committee. Results: The percentage resistance towards carbapenems in E.coli, Klebsiella, Pseudomonas and A. baumannii from July 2007-June 2008 was 1.07, 13.1, 21.3 and $12.5 \%$ respectively. Phase I intervention programme was initiated in July 2008 and Phase II in July 2010 and a subsequent reduction of $4.03 \%$ was observed in the carbapenem resistant Pseudomonas in the last stage of study period following the interventions. However the resistance in the other Gram negatives (E. coli, Klebsiella and A. baumannii) rose and then stabilized. Conclusion: An antimicrobial stewardship programme with sustained and multifaceted efforts is essential to control the increasing resistance of microorganisms towards antibiotics.
\end{abstract}

Key words: Carbapenem resistance, gram negatives, antimicrobial stewardship program

\section{INTRODUCTION}

Antimicrobial resistance is not a new phenomenon; however, the current magnitude and the speed with which it is developing is a cause for global concern including our country. Studies and surveys indicate that as much as half of all antimicrobial use is inappropriate. Antimicrobial resistance not only increases mortality and morbidity in healthcare but also leads to spiraling healthcare costs adversely affecting trade and economies. The threat of the post antibiotic era looms ahead (GARP, 2011). Antimicrobial agents have been used in human medicine for more than 50 years, with tremendous benefits to health. However, because resistance to antimicrobial drugs is expected to occur with their prolonged use, it is essential that such drugs be regulated and used judiciously to delay the development of resistance. Misuse and overuse of these drugs contribute to an even more rapid development of resistance. After several decades of successful antimicrobial use, the emergence of multi-resistant bacteria pathogens, which are less responsive to therapy, is observed. Till now, Extended Spectrum $\beta$ Lactamase (ES $\beta \mathrm{L})$ production by Gram negative bacteria particularly in Enterobacteriaceae was considered as the most important threat to clinical therapeutics (Livermore, 1998; Mathur et al., 2002; Paterson, 2006).

Therapy with a carbapenem has historically been the agent of choice for empiric therapy of infections caused by Acinetobacter and other Gram negatives. However, resistance to carbapenems is increasing

Corresponding Author: Namita Jaggi, Department of Lab Services and Infection Control, Artemis Health Institute, Sector 51, Gurgaon, Haryana Tel: +91-9811323628 Fax: 0124-6767701 
(Baumgart et al., 2010). It is acquired mainly due to acquisition of genes responsible for expression of Metallo-Beta-Lactamases (MBLs) producing enzymes. This not only renders the bacteria resistant to carbapenems but also confers high-level resistance to all $\beta$-lactams except aztreonam involving various host organisms, most commonly Enterobacteriaceae, Pseudomonas and Acinetobacter. The need of the hour is to develop a robust antimicrobial stewardship programme which would enhance clinical outcomes, reduce healthcare costs and minimize adverse effects of antimicrobial use (toxicity and resistance).

We conducted this retrospective analysis for a period of four years (July 2008 to June 2011) to look for the antimicrobial activity of carbapenems in Gramnegative bacilli isolated from various clinical specimens in a tertiary care hospital in India.

\section{MATERIALS AND METHODS}

The culture data of all samples was analyzed for a period of four years (July 2007 to June 2011) in the Microbiology lab of a tertiary care hospital. The various specimens obtained from the hospital included urine, blood, respiratory (sputum, tracheal aspirate and broncho-alveolar Lavage), sterile body fluid, pus and stool specimens. Standard culture methods were used and the isolates, both Gram positive and Gram negative were processed for identification and antibiotic sensitivity tests by the Vitek 2 Compact system (bioMerieux, Marcy l'Etoile, France), following CLSI guidelines (CLSI, 2007; 2009; 2011). The antibiogram of each confirmed isolate was studied and susceptibility results were compiled with the WHONET 5.4 programme.

\section{Our study was staged into four parts:}

July 2007 to June 2008: The antimicrobial resistance data of the hospital isolates from various samples was retrospectively analyzed over a period of one year (July 2007 to June 2008). Resistance patterns of the most common Gram negative isolates E.coli, Klebsiella, Pseudomonas and Acinetobacter baumannii were studied to determine the percentage of resistance towards carbapenems.

July 2008 to Dec 2008: Phase I intervention programme: In July 2008, the Phase I intervention programme was introduced in which the formulation and implementation of an antibiotic policy in the hospital was done. This dealt with Empiric antibiotic policy, Pre-surgical prophylaxis and Reserve drug antibiotic policy. It was formulated on the basis of CDC guidelines and the local antibiotic data and prescribing preferences. For this, a detailed discussion with every clinician took place and the doctors' preferences were incorporated in this policy.

Jan 2009 to June 2010: The Impact of Phase I intervention programme was assessed in the following year (2009) and the first half of 2010 in terms of antibiotic resistance percentage of the Gram negative isolates similar to the first year.

July 2010 to June 2011: Phase II intervention programme: In July 2010 an antimicrobial stewardship programme was initiated which included the constitution of the Antimicrobial Stewardship committee. Its primary aim was to optimize clinical outcome and minimize unintended consequences of antimicrobial use namely toxicity and selection of drug resistant pathogens. It was secondarily aimed to reduce healthcare costs. The existing antibiotic guidelines were re-examined and modified depending on the antibiograms and discussion with physicians. Prospective audit with intervention and feedback and formulary restriction and preauthorization were also followed. Rigorous infection control policies and protocols were followed to prevent the spread of multiresistant pathogens.

The impact of Phase II intervention was observed in the following year with respect to carbapenem resistance of Gram negative isolates.

\section{RESULTS}

Of the 31414 samples received in the microbiology laboratory of a tertiary care hospital during the study period of 48 months (July 2007 to Jun 2011), there were 11961 urine samples, 10244 blood samples, 4041 respiratory samples (sputum, tracheal aspirates and bronchoalveolar lavage), 1908 sterile body fluids, 1769 pus and 1491 stool samples (Table 1). Urine cultures were the predominant sample type comprising of 11961 samples out of a total of 31414 (38.07\%).

There were a total of 6160 isolates obtained from 31414 cultures $(19.6 \%)$. Out of the total isolates 5007 were Gram negative showing a clear preponderance of Gram negative pathogens in the hospital environment (5007 of $6160,81.2 \%)$. Among the Gram negatives E.coli (44.1\%), Klebsiella (20.2\%), Pseudomonas $(15.05 \%)$ and A. baumannii $(9.7 \%)$ were the predominant isolates.

According to our staged intervention plan, the resistance data of Gram negative isolates (E.coli, Klebsiella, Pseudomonas and A. baumannii) was retrospectively analyzed over the period of one year (July 2007 to June 2008). The results are displayed in Table 2. The percentage resistance towards carbapenems in E.coli, Klebsiella, Pseudomonas and A. baumannii from July 2007-June 2008 was 1.07, 13.1, 21.3 and $12.5 \%$ respectively. 
Am. J. Infect. Dis., 8 (2): 106-111, 2012

Table 1: Total cultures from July 2007 to June 2011

\begin{tabular}{|c|c|c|c|c|c|c|}
\hline Period & $\begin{array}{l}\text { Total } \\
\text { samples }\end{array}$ & Break-up & & $\begin{array}{l}\text { Total } \\
\text { isolates }\end{array}$ & $\begin{array}{l}\text { Gram negative } \\
\text { isolates }(\%)^{*}\end{array}$ & $\begin{array}{l}\text { Gram positive } \\
\text { isolates (\%)* }\end{array}$ \\
\hline \multirow[t]{7}{*}{ July 2007 - Jun 2011} & 31414 & Urine & 11961 & 2342 & $2016(86.0)$ & $326(13.91)$ \\
\hline & & Blood & 10244 & 997 & $733(73.5)$ & $264(26.4)$ \\
\hline & & Respiratory & 4041 & 1312 & 1202 (91.6) & $110(8.3)$ \\
\hline & & Pus & 1769 & 948 & $571(60.02)$ & 377 (39.7) \\
\hline & & Stool & 1491 & 380 & $380(100)$ & 0 \\
\hline & & Fluids & 1908 & 181 & $105(58.01)$ & 76 (41.9) \\
\hline & & & 31414 & $6160(19.6 \%)$ & $5007(81.2 \%)$ & $1153(18.7 \%)$ \\
\hline
\end{tabular}

*: Percentage out of Total Isolates

Table 2: Carbapenem Resistance in Gram negatives from July 2007 to Jun 2011

\begin{tabular}{|c|c|c|c|c|c|c|c|c|}
\hline \multirow[b]{2}{*}{ Period } & \multicolumn{2}{|c|}{ E.coli } & \multicolumn{2}{|c|}{ Klebsiella } & \multicolumn{2}{|c|}{ A. baumannii } & \multicolumn{2}{|c|}{ Pseudomonas } \\
\hline & $\mathrm{n}^{*}$ & Carb®\# (\%) & $\mathrm{n}^{*}$ & Carb®\# (\%) & $\mathrm{n}^{*}$ & Carb®\# $(\%)$ & $\mathrm{n}^{*}$ & Carb®\# (\%) \\
\hline July 2007-June 2008 & 278 & $3(1.07)$ & 61 & $8(13.1)$ & 16 & $2(12.5)$ & 89 & $19(21.3)$ \\
\hline July 2008- Dec 2008 & 253 & $9(3.6)$ & 108 & $31(29)$ & 27 & $17(63)$ & 79 & $10(12.6)$ \\
\hline Jan 2009- Dec 2009 & 601 & $22(3.67)$ & 371 & $108(29.1)$ & 136 & $121(88.9)$ & 294 & $97(32.9)$ \\
\hline Jan 2010- Jun 2010 & 320 & $14(4.38)$ & 132 & $35(26.5)$ & 102 & $89(87.2)$ & 86 & $30(34.8)$ \\
\hline July 2010- June 2011 & 759 & $46(6.06)$ & 343 & $99(28.8)$ & 205 & $188(91.7)$ & 206 & $69(33.4)$ \\
\hline
\end{tabular}

*: Total no. of isolates \#: Carbapenem resistant isolates

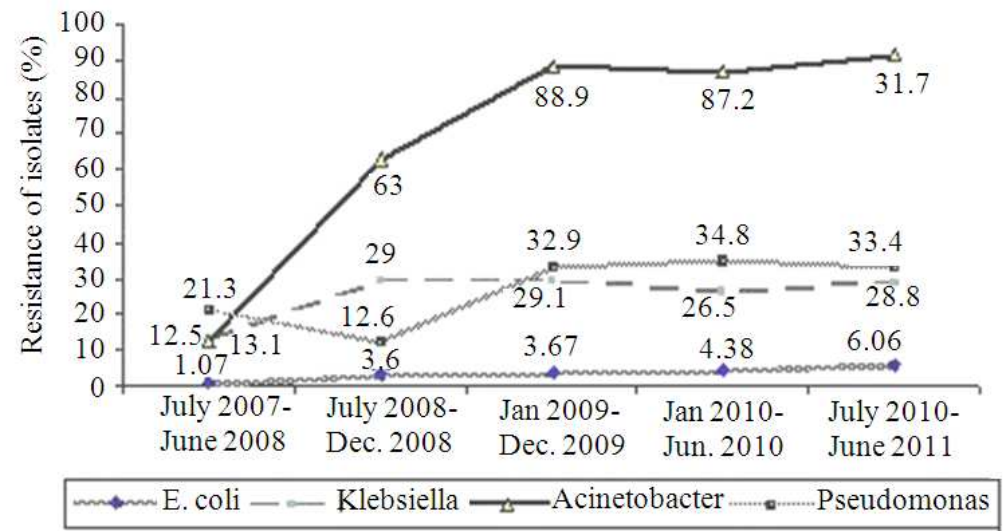

Fig. 1: Carbapenem resistance in gram negatives

Phase I intervention programme was initiated in July 2008.The carbapenem resistance in Pseudomonas reduced to $12.6 \%$ in the first six months period (July 2008 to Dec 2008). However the resistance in the other Gram negatives E.coli, Klebsiella and A. baumannii rose to $3.6,29$ and $62.9 \%$ respectively. In the subsequent year (Jan 2009 to Dec 2009), the carbapenem resistance in E.coli and Klebsiella showed stable figures of 4 and $29.1 \%$ respectively. However in A. baumannii and Pseudomonas the resistance showed significant increase to 88.9 and $32.9 \%$ respectively. The trend of decreasing resistance continued in the next six months (Jan 2010-June 2010) for Klebsiella and A. baumannii as 26.5 and $87.2 \%$ respectively but E.coli and Pseudomonas witnessed a slight increase of 4.38 and $34.8 \%$ respectively.

Upon the introduction of Phase II intervention programme in July 2010, Pseudomonas showed a further decrease in carbapenem resistance $(33.4 \%)$ in the following year (July 2010 to June 2011). However, the percentage of carbapenem resistance in E coli, Klebsiella and A. baumannii rose to 6.06, 28.8 and $91.7 \%$ respectively.

Following this programme a significant decrease of $4.03 \%$ was only observed in case of carbapenem resistant Pseudomonas. The rest of the resistance patterns were somewhat stable possibly indicating a more prolonged time period required to have an 
impact or the natural evolution of antimicrobial resistance (Fig. 1).

\section{DISCUSSION}

There are a large number and variety of new resistance mechanisms that have emerged and their preliminary detection is important for infection control and adequate therapeutic guidance.

In our study, the impact of Antimicrobial Stewardship Programs run in two phases (Phase I in July 2008 and Phase II in July 2010) was evaluated with respect to the carbapenem resistance in Gram negatives E.coli, Klebsiella, Pseudomonas and A. baumannii. Reviews by MacDougall and Polk (2005) and Fishman (2006) have also summarized similar studies on impact of stewardship interventions on antimicrobial use.

The first phase was somewhat successful in reducing the carbapenem resistance only in Pseudomonas. However, the resistance percentage increased in E.coli, Klebsiella and A. baumannii to 3.6, 29 and $62.9 \%$ where $A$. baumannii showed the highest increase in resistance (from 21.3-62.9\%). This could be because of entry of MDR A. baumannii from outside as the infection control practices and protocols were followed religiously in the hospital. Also it indicated that perhaps there was continuing misuse of carbapenems. Studies suggest that the increase in Resistance of Pseudomonas and A. baumannii to carbapenems may result from changes in the penicillinbinding proteins and porins. Since carbapenems enter the bacterium through the porins, one could postulate that porins changes could be involved in the increasing resistance to these antibiotics (Nordmann and Poirel, 2002; Oliver, 2004; Poirel and Nordmann, 2006). This explains the reason behind continuous increase of carbapenem resistance in Pseudomonas and $A$. baumannii to 32.9 and $88.9 \%$ respectively in the following year (2009) as the other Gram negatives E.coli and Klebsiella showed stable figures of resistance( 4 and $29.1 \%$ respectively).

There was a decrease in resistance observed in first half of 2010 (Jan 2010 to June 2010) for Klebsiella and A. baumannii as 26.5 and $87.2 \%$ respectively but E.coli and Pseudomonas witnessed a slight increase (34.8 and $4.38 \%)$. Under this situation of overall increasing resistance, it was felt that a formalized Antimicrobial stewardship committee be formulated and a boost given to the antimicrobial stewardship programme as the impact of the Phase I intervention was waning. This resulted in the introduction of the Phase II intervention program in July 2010 which involved the constitution of the Antimicrobial Stewardship committee. The function of this committee was to optimize clinical outcome and minimize unintended consequences of antimicrobial use namely toxicity and selection of drug resistant pathogens, modify existing antibiotic guidelines as required depending on the antibiograms and discussion with physicians. It also included in its key roles Prospective audit with intervention and feedback and formulary restriction and preauthorization which were implemented in combination with rigorous infection control policies and protocols to prevent the further spread of multi-resistant pathogens. It was aimed to secondarily reduce healthcare costs. As recommended by Patel et al. (2008), the Antimicrobial Stewardship interventions include prospective audit and intervention, formulary restriction, education, guideline development, clinical pathway development, antimicrobial order forms and the de-escalation of therapy.

The impact of this program was observed in the next year (July 2010 to June 2011) in Pseudomonas as decrease in carbapenem resistance percentage (33.4\%) was observed. However, all the other Gram negatives (E coli, Klebsiella and A. baumannii) showed a significant increase in resistance as rose to $6.06,28.8$ and $91.7 \%$ respectively.

In our study Pseudomonas showed varying degrees of resistance towards carbapenems. The rest of the resistance patterns were somewhat stable possibly indicating a prolonged time period required to have an impact or the natural evolution of antimicrobial resistance. The resistance to carbapenems especially in Pseudomonas results from reduced levels of drug accumulation or increased expression of pump efflux12. The resistance may also be due to the production of Metallo-B-Lactamases (MBL) which can be chromosomally encoded or plasmid mediated (Navaneeth et al., 2002; Hancock, 1998). Our results are supported by Forster and Daschner (1998) and Gonlugur et al. (2004) in their studies showing varying resistance (4-60\%) of Pseudomonas towards these drugs across the World.

The most recent investigation regarding the successful control of the hypervirulent strain of $C$. difficile used a combination of antimicrobial interventions and enhanced infection control (Muto et al., 2007). Nonetheless, antimicrobial stewardship programs are associated with clear value and will be increasingly an integral part in the inpatient healthcare setting. National and international organizations have recognized the growing problem of antimicrobial resistance and have published recommendations to combat this problem (Brown and Nathwani, 2005; DH, 2000; WHO, 2001). Although resistance is a worldwide concern, it is first and foremost a local problem: selection for and amplification 
of resistant members of a species are occurring in individual hospitals (and communities), which can then spread worldwide (O’Brien, 1997; 2002).

\section{CONCLUSION}

In conclusion, our study highlights the increasing resistance in Gram-negative bacteria towards carbapenems in our hospital. As our study was limited to the carbapenem resistant bacteria isolated from patients in a tertiary care hospital, true extent of resistance to these agents among bacterial isolates from community acquired infections may be considerably low. In this study, though we have initiated an antimicrobial stewardship program and have started conducting audits, we have not vey stringently looked at the dosages and pharmacodynamics and pharmacokinetics. In addition, regular antimicrobial susceptibility surveillance and a comparison of defined daily dosages with the antimicrobial resistance are essential to evaluate the actual outcome of an antimicrobial stewardship program. There is also a need to emphasize on the rational use of antimicrobials and strictly adhere to the concept of "reserve drugs" to minimize the misuse of available antimicrobials.

\section{ACKNOWLEDGEMENT}

We owe our great thanks to Artemis Health Institute for extending their kind support as a funding source and encouraging us in writing this study.

There exists no potential conflict of interest in terms of financial and other relationships and this is applicable to all the three authors (Namita Jaggi, Pushpa Sissodia and Lalit Sharma).

\section{REFERENCES}

Baumgart, A.M., M.A. Molinari and A.C. Silveira, 2010. Prevalence of carbapenem resistant Pseudomonas aeruginosa and Acinetobacter baumannii in high complexity hospital. Braz. J. Infect. Dis., 14: 433-436. PMID: 21221469

Brown, E.M. and D. Nathwani, 2005. Antibiotic cycling or rotation: A systematic review of the evidence of efficacy. J. Antimicrob. Chemother., 55: 6-9. DOI: $10.1093 / \mathrm{jac} / \mathrm{dkh} 482$

CLSI, 2007. Performance standards for antimicrobial susceptibility testing; seventeenth informational supplement. Clinical and Laboratory Standards Institute, Wayne, PA.

CLSI, 2009. Performance standards for antimicrobial susceptibility testing; nineteenth informational supplement. Clinical and Laboratory Standards Institute, Wayne, PA.
CLSI, 2011. Performance standards for antimicrobial susceptibility testing; twenty-first informational supplement. Clinical and Laboratory Standards Institute, Wayne, PA.

$\mathrm{DH}, 2000$. UK antimicrobial resistance strategy and action plan. Department of Health.

Fishman, N., 2006. Antimicrobial stewardship. Am. J. Med., 119: S53-S61. PMID: 16735152

Forster, D.H. and F.D. Daschner, 1998. Acinetobacter Species as Nosocomial Pathogens. Eur. J. Clin. Microbiol Infect. Dis., 17: 73-77.

GARP, 2011. Rationalizing antibiotic use to limit antibiotic resistance in India. Indian J. Med. Res., 134: 281-294.

Gonlugur, U., M.Z. Bakici, I. Akkurt and T. Efeoglu, 2004. Antibiotic susceptibility patterns among respiratory isolates of Gram-negative bacilli in a Turkish university hospital. BMC Microbiol., 4: 32-32. DOI: $10.1186 / 1471-2180-4-32$

Hancock, R.E., 1998. Resistance mechanisms in Pseudomonas aeruginosa and other nonfermentative gram-negative bacteria. Clin. Infect. Dis., 27: S93-S99. PMID: 9710677

Livermore, D.M., 1998. Beta-lactamase-mediated resistance and opportunities for its control. J. Antimicrob. Chemother., 41: 25-41. DOI: 10.1093/jac/41.suppl_4.25

MacDougall, C. and R.E. Polk, 2005. Antimicrobial stewardship programs in health care systems. Clin. Micro. Rev., 18: 638-656. DOI: 10.1128/CMR.18.4.638-656.2005

Mathur, P., A. Kapil, B. Das and B. Dhawan, 2002. Prevalence of extended spectrum beta lactamase producing gram negative bacteria in a tertiary care hospital. Indian J. Med. Res., 115: 153-157. PMID: 12239838

Muto, C.A., M.K. Blank, J.W. Marsh, E.N. Vergis and M.M. O'Leary et al., 2007. Control of an outbreak of infection with the hypervirulent Clostridium difficile BI strain in a university hospital using a comprehensive "bundle" approach. Clin. Infect. Dis., 45: 1266-1273. PMID: 17968819

Navaneeth, B.V., D. Sridaran, D. Sahay and M.R.S. Belwadi, 2002. A preliminary study on metallo blactamase producing Pseudomonas spp. aeruginosa in hospitalized patients. Indian J. Med. Res., 116: 264-267.

Nordmann, P. and L. Poirel, 2002. Emerging carbapenemases in Gram-negative aerobes. Clin. Microbiol. Infect., 8: 321-331. DOI: 10.1046/j.1469-0691.2002.00401.x 
Am. J. Infect. Dis., 8 (2): 106-111, 2012

O'Brien, T.F., 1997. The global epidemic nature of antimicrobial resistance and the need to monitor and manage it locally. Clin. Infect. Dis., 24: S2-S8. PMID: 8994775

O'Brien, T.F., 2002. Emergence, spread and environmental effect of antimicrobial resistance: how use of an antimicrobial anywhere can increase resistance to any antimicrobial anywhere else. Clin. Infect. Dis., 34: S78-S84. DOI: 10.1086/340244

Oliver, A., 2004. Resistencia a carbapenemas y Acinetobacter baumannii. Enferm. Infec. Microbiol. Clin., 22: 259-261.

Patel, D., W. Lawson and B.J. Guglielmo, 2008. Antimicrobial stewardship programs: Interventions and associated outcomes. Expert Rev. Anti. Infect. Ther., 6: 209-222. DOI: 10.1586/14787210.6.2.209
Paterson, D.L., 2006. Resistance in gram-negative bacteria: Enterobacteriaceae. Am. J. Med., 119: S20-S28. PMID: 16735147

Poirel, L. and P. Nordmann, 2006. Carbapenem resistance in Acinetobacter baumannii: Mechanisms and epidemiology. Clin. Microbiol. Infect., 12 : $826-836$. DOI: $10.1111 / j .1469-$ 0691.2006.01456.x

WHO, 2001. WHO global strategy for containment of antimicrobial resistance. World Health Organization, Geneva, Switzerland. 